\title{
Study of the Health Instructions Effect on Quality of Life and Psychological Problems among Children with Thalassemia
}

\author{
Seham Mohammed Elmwafie Hassan ${ }^{1}$ \& Saida El Sayed Hassan Ibrahim El Azzab ${ }^{2}$ \\ ${ }^{1}$ Lecturer of pediatric Nursing, Faculty of Nursing, Beni-Suef University, Egypt \\ ${ }^{2}$ Lecturer of Psychiatric/Mental Health Nursing, Faculty of Nursing, Beni-Suef University, Egypt \\ Correspondence: Seham Mohammed Elmwafie Hassan, Lecturer of pediatric Nursing, Faculty of Nursing, \\ Beni-Suef University, Egypt.
}

Received: November 5, 2016

Accepted: November 26, 2016

Online Published: December 9, 2016

doi:10.20849/ijsn.v1i1.92

URL: http://dx.doi.org/10.20849/ijsn.v1i1.92

\begin{abstract}
Objective: The aim of this study was to evaluate the health instructions effect on quality of life and psychological problems among children with thalassemia.

Design: Quasi-experimental design used in this study. Pre and post evaluation done to identify the effect of health instructions sessions which provided by the researchers for thalassemic children and their parents on children heath related quality of life and psychological problems using Pediatric quality of life inventory PedsQL 4.0 generic core scale which was used to measure health-related quality of life in children and adolescent. In addition, Depression, Anxiety and Stress Scale (DASS) used to evaluate psychological problems among children.
\end{abstract}

Setting: It was conducted at outpatient and hematological unit affiliated to Beni-Seuf University hospital.

Sample: The total children included in this study were 23 children of either sex, aged (7-18y). Children with physical or mental handicapped excluded from the study.

Results: The results of this study showed that more than two third of studied children scored medium level in physical and school function. As well as, there was no significant differences in thalassemic children health related quality of life domains post health instructions sessions where $\mathrm{p}>0.05$. Also these study findings demonstrated that $60 \%$ of children had extremely sever anxiety and $43.5 \%$ of them had severe depression. However, post health instruction sessions these findings showed statistical significant decline where $\mathrm{p}$ value $=.001$ and .000 respectively.

Conclusion: An important finding to emerge in this study is studied children with thalthemia had psychological problems with poorer health related quality of life. However, the results set out to the provision of health instructions for children with thalthemia and their parents had significant effect in improvement of children psychological problems. Unfortunately, there is no significant effect in their health related quality of life post instructions sessions. Thus, the results of this study suggest that the importance for the continuous heath educations program providing for thalassemic children and their parents by pediatric and psychiatric nurses. In addition, health team collaboration for monitoring psychological problems of children with thalassemia and assist children and their parents to more adjustment in their health related quality of life.

Keywords: thalassemia, psychological problems, quality of life, health instructions

\section{Background and Introduction}

Worldwide, thalassaemia poses a serious public health problem due to the high prevalence extending from the Mediterranean basin and parts of Africa, throughout the Middle East, the Indian Sub-continent, and into South-East Asia. Its' global prevalence is about 2 per 1,000 newborns and 50,000 to 100,000 children die of thalassaemia major in low and middle income countries while about $7 \%$ of the world's population is a carrier of a hemoglobin disorder (World Health Organization, 2008; Baghianimoghadam et al., 2011). Thalassaemia consists one of the most common chronic and genetic hematological disorder, globally. There are approximately 240 million people worldwide who are heterozygous for $\beta$-thalassemia while approximately 200,000 affected homozygotes are born annually (Wong et al., 2011). 
In the Egypt, occurrence of thalassemia disorders is increasing. In Egypt carrier rate $5.3-\geq 9 \%$, and 1000/1.5 million / year live births born with thalassemia (total live births 1,936,205 in 2006). Registered cases of homozygous beta thalassemia in big centers of Egypt in 2006 up to Sept 2007 (n=9912) (El-Beshlawy, 2009). There are 10,000 registered thalassemia cases and more than 20,000 non-registered cases. $95 \%$ are beta thalassemia major and 5\% are thalassemia intermediate or hemoglobin $\mathrm{H}$ disease (Mahmoud, 2009)

Thalassemia is a major health problem not only for the patients and their families but also for the Public Health System of each country due to the cost of treatment involving regular blood transfusions, iron chelation, frequent hospitalization and general medical follow up. That creates a burden not only on health system but also on the affected families, and children, which become vulnerable to emotional, social, psychological and behavioral problems (Shaligram et al., 2007; Wong et al., 2011).

The impact of the disease in many dimensions of life becomes increasingly evident during the school age and adolescent when children seek for independence. The treatment modalities and the disease itself may have profound impact on the patients' health-related quality of life (HRQOL) which include physical appearance, such as bone deformities and short stature which leads to poor self-image (Ismail et al. 2006; Telfer et al. 2005). As well as, it has associated with psychosocial aspect and a significant negative impact on areas of school functioning. Thalassemic children have been described to show impaired abstract reasoning, deficits of language, attention, memory, visual spatial skills, and executive functions, all of which are more prominent in hemosiderotic subjects (Monastero et al., 2000). In most children, low intelligence quotient appears to be correlated with poor school performances and physical or social restrictions (Economou et al., 2006). Therapy of thalassemia includes a regular monthly blood transfusion, however, patients will inevitably confront with several side effects particularly iron overload in body critical organs and tissues including heart, ductless glands and liver (Azarkeivan, et al., 2009; Roy\&Chatterjee, 2007 ; Indaratna, et al., 2010).

In addition, children with thalassemia face many emotional challenges (Chaturvedi, et al., 2007). Mood swings quite abruptly in children with thalassemia is very natural and a major challenge. They may feel confidence in the future in one day of a week but after that day, they may feel nervous, restless and hopeless. Feeling good gives them power to lead a normal life. On the other hand, feeling hopeless undermines their willpower to fight with the disease. Children with thalassemia have to deal with emotions such as fear of needles, pain and anger (Borgna et al., 2010).

Thus, children with thalassaemia and their parents require lifelong psychological support for prevention of mental health issues. Several effective psychological strategies is include counseling and given information for families and children can be an effective approach to children with thalassaemia major, capable of increasing compliance to treatment, lessening the emotional burden of disease and improving the quality of life of children and their caregivers (Mazzone et al, 2009). The awareness of the illness has been suggested as a possible factor influencing both the compliance with treatment and the quality of life of children and their caregiver. In fact, children and their parents, who were more conscious of the illness, were also more compliant with the therapy (Aydinok et al., 2005).

Since, inappropriate coping behaviors causes psychological-mental problems in parents and following them, in their children, the necessity of implementation of nursing interventions in this field and doing the individual and group consulting of parents seem absolutely necessary. Nurses and other caregivers in the health-treatment team should focus on the programs and intervention that can improve the coping behaviors of parents and their children with thalassemia and or other chronic diseases and use these programs in care planning for these patients (Salehi\& Morovati, 2016).The objectives of the nursing care for children and adolescent with thalassemia start with promote adherence to treatment regimen, as well as support them during illness and distressing treatments, in addition promote patient and family coping such as anticipate older children and adolescents concerns related to appearance and monitor closely for complications of the condition and treatment (Johnson, 2010).

\subsection{Significance of the Study}

Until now children with thalassemia and their families who care face many challenges. Health related quality of life aspect and psychological problems of children with thalassemia was dim and ignored, we tried to highlight this area especially in the rural area where resources are not so feasible aiming at improving children' HRQOL in non-costly ways, such as offering counseling, health instruction and psychological support. As there are limited studies in Egypt focusing on thalassaemic children psychological needs and HRQOL, and in order to explore health instruction affects on children psychological improvement. 


\section{Research Aim}

The aim of this research was study of the health instructions effect on quality of life and psychological problems among children with thalassemia. We hypothesized that health instructions provided by nurses for children with thalassemia and their parents would effect on the psychological problems of children and improving their heath related quality of life.

\section{Material and Methods}

This study used quasi-experimental design. The study was conducted at outpatient and hematological unit affiliated to Beni-Seuf University hospital it serves a large number of thalathemic children. Convenience, purposeful sample was used in this study, the total children included in this study were 23 children of either sex, aged (7-18y). Children with physical or mental handicapped excluded from the study. Tools used for data collection were the following; the first tool was the socio -demographic and clinical data questionnaire developed by the researchers to collect demographic and clinical data such as age, gender, education, frequency of blood transfusion, family past history and duration of illness. The second tool was pediatric quality of life inventory (PedsQL) 4.0 generic core scale which was developed to measure health-related quality of life (HRQOL) in children and adolescent with acute and chronic health problems. This Scale provided by (James, 2006) at MAPI Research Institute in Lyon, France, The Arabic forms used included parallel child self reports (age ranges 5-7, 8-12, and 13-18 years). PedsQL items required subjects to report problems regarding physical, emotional, social and schooling function that have arisen for the past one month. The 23 items' responses were measured on a five-point rating scale ranging from 0 (never a problem) to 4 (almost always a problem. Each scale had a score ranging from 0 to 100, the higher score indicating higher HRQOL. As well as, the third tool used was the Depression, Anxiety and Stress Scale (DASS) which developed by (Taouk et al., 2008) and used to measure psychological problems among studied children with thalassemia. Scoring and grading of DASS scale included in as the following.

\begin{tabular}{llll} 
Category & \multicolumn{3}{l}{ Depression Anxiety Stress } \\
Normal & $0-9$ & $0-7$ & $0-14$ \\
Mild & $10-13$ & $8-9$ & $15-18$ \\
Moderate & $14-20$ & $10-14$ & $19-25$ \\
Severe & $21-27$ & $15-19$ & $26-33$ \\
Extremely severe & $28+$ & $20+$ & $34+$
\end{tabular}

\subsection{Validity and Reliability}

The PedsQL has demonstrated good internal consistency and validity. Internal consistency reliability for the total Scale Score alpha $=0.88$. In addition, Depression, Anxiety and Stress Scale (DASS) to evaluated psychological problems among children. It is a 42-item self-report inventory designed to measure the presence and severity of symptoms of depression, anxiety and stress among young children. This scale was psychometrically validated to the Arabic culture. Gamma coefficients that represent the loading of each scale on the overall factor (total score) are 0.71 for depression, 0.86 for anxiety, and 0.88 for stress

\subsection{Field Work Descriptions}

The overall study work carried out through May 2015 and continued to August 2016, during which time children with thalathemia and their parents were interviewed with self - permission to participate in the study sequentially on their scheduled appointments at the Hematology Clinic and blood transfusion time. The study was conducted through two phases, phase one was carried out to collect socio demographic data, apply DAAS scale and PedsQL to determine psychological and HRQOL of children with thalassemia before any health instruction this phase took 4 months. Then start health instruction sessions according to their individual needs, after 10 sessions for every child and their parent which took 7 months of instruction. The second phase started to apply the same scaled for analyzing the differences.

Based on the health instruction intervention plan, the researchers were present in the hematological clinic and unit at three days / week. The health instructions afforded according to health needs such as nutrition, methods to relive pain, compliance with treatment plan, blood transfusion importance and follow up schedule. In each session we discussed with children and their families their feelings and unsolved problems which were usually 
occurred in their life and related to thalathemia as a chronic disease. Then, the researcher tried to change these concern regarding problems with counseling and given guidance and instruction with the advice of medical sense Provision of information was individualized and appropriate to the age, developmental stage personality and family environment. We have included caregivers in health instruction sessions to improve their knowledge, enhancing communication, and ultimately to promote a greater compliance to thalassaemia therapy and guidance and to improve the quality of their life.

\subsection{Limitations}

This study has the following limitations: it was performed exclusively in the Beni - Suef City hence, its results exclusively reflect the small group of children and adolescents. In addition, many patients did not come regularly for follow up and blood transfusion so lead to more time in completes this study.

\section{Data Analysis}

Statistical analysis was conducted using the Statistical Package for the Social Sciences (SPSS, Version 14 for Windows). The significance of differences was tested using the $\chi^{2}$ test and ANOVA at the $5 \%$ level of probability significance. Total HRQOL score and summary scores were presented in terms of mean and standard deviation. $\mathrm{P}$-value of $\leq 0.05$ was considered statistically significant.

\section{Ethics Approval}

This study was granted ethical approval from the head of pediatric department. As well as the oral consent taken from children and their care givers after explaining them the aim of the study.

\section{Results}

Table 1. Socio- demographic characteristics and clinical data of studied children

\begin{tabular}{|c|c|c|}
\hline Variables & Frequency $(n=23)$ & Percentage (100\%) \\
\hline \multicolumn{3}{|l|}{ Age (in years) } \\
\hline $7-11$ & 15 & 65.2 \\
\hline \multirow[t]{2}{*}{$\geq 12$} & 8 & 34.8 \\
\hline & \multicolumn{2}{|c|}{ Mean $\pm \mathrm{SD}=9.95 \pm 3.62$} \\
\hline \multicolumn{3}{|l|}{ Gender } \\
\hline Male & 15 & 65.2 \\
\hline Female & 8 & 34.8 \\
\hline \multicolumn{3}{|l|}{ Study phase } \\
\hline Primary level & 9 & 39.1 \\
\hline Prep-school & 10 & 43.5 \\
\hline Secondary level & 4 & 17.4 \\
\hline \multicolumn{3}{|c|}{ Thalassaemia family history } \\
\hline No & 8 & 34.8 \\
\hline Yes & 15 & 65.2 \\
\hline \multicolumn{3}{|c|}{ Mother education } \\
\hline Illiterate & 10 & 43.5 \\
\hline Read and write & 4 & 17.4 \\
\hline$\geq$ Secondary & 9 & 39.1 \\
\hline \multicolumn{3}{|c|}{ Father education } \\
\hline Illiterate & 5 & 21.7 \\
\hline Read and write & 8 & 34.8 \\
\hline$\geq$ Secondary & 11 & 43.5 \\
\hline \multicolumn{3}{|c|}{ Length of diagnosis } \\
\hline$\leq$ year & 5 & 21.7 \\
\hline$<$ year -5years & 15 & 65.2 \\
\hline$>5$ years & 3 & 13.1 \\
\hline \multicolumn{3}{|c|}{ Blood transfusion Frequency } \\
\hline Once / month & 6 & 26.1 \\
\hline Once/2 month & 5 & 21.7 \\
\hline Once/6month & 8 & 34.8 \\
\hline None & 4 & 17.4 \\
\hline
\end{tabular}


Table 1: As regards socio-demographic characteristics of the studied children Table 1 obvious that, the more than two third of them $(65.2 \%)$ their age ranged between 7 to 11 years old, with mean age $9.95 \pm 3.62$ years and out of total 23 patients, 15 were males. Concerning their study phase, this table point out that $43.5 \%$ of them prep-school and $17.4 \%$ of them at secondary phase. As regard children' fathers and mothers educational level, it was evident that $43.5 \%, 39.1 \%$ have a $\geq$ secondary educational level respectively. Family history of thalassaemia was positive in $65.2 \%$ of children, as well as, $65.2 \%$ of them diagnosed with thalassaemia from year to 5 years. While, $26.1 \%$ of them required blood transfusions once each month with nearly one third $34.8 \%$ of them reported once each 6 months.

Table 2: Concerning the health related quality of life of studied children pre and post health instruction, this table illustrates that, more than two third of patients (65.2\%) scored medium grade regarding their physical functioning. While $69.6 \%$ of them scored medium post health instructions sessions with no statistical significant difference where $p=.714$. As well as, nearly one third (39.1\% and $30.4 \%)$ of them scored good social function pre and post health instruction respectively. This table also shows that, no statistical significant difference pre and post health instructions seen for the emotional and school function scores where $\mathrm{P}=.833, .328$ respectively.

Table 2. Mean differences between children scores on the quality of life pre and post health instruction

\begin{tabular}{|c|c|c|c|c|c|c|c|c|}
\hline \multirow[t]{2}{*}{ Grade of QOL } & \multicolumn{3}{|c|}{ Pre-instruction } & \multicolumn{3}{|c|}{ post-instruction } & \multirow{2}{*}{$\mathbf{t}$} & \multirow{2}{*}{ p-value } \\
\hline & No & $\%$ & Mean \pm SD & No & $\%$ & Mean \pm SD & & \\
\hline \multicolumn{9}{|l|}{ Physical } \\
\hline Low & 2 & 17.4 & & 3 & 13.0 & & & \\
\hline Medium & 15 & 65.2 & $50.00 \pm .15 .07$ & 16 & 69.6 & $51.04 \pm 14.05$ & 371 & .714 \\
\hline Good & 4 & 17.4 & & 4 & 17.4 & & & \\
\hline Perfect & 0 & 0.0 & & 0 & 0.0 & & & \\
\hline \multicolumn{9}{|l|}{ Social } \\
\hline Low & 7 & 30.4 & & 7 & 30.4 & & & \\
\hline Medium & 3 & 13.0 & $60.43 \pm 28.12$ & 5 & 21.7 & $58.34 \pm 28.13$ & .810 & .426 \\
\hline Good & 9 & 39.1 & & 7 & 30.4 & & & \\
\hline Perfect & 4 & 17.4 & & 4 & 17.4 & & & \\
\hline \multicolumn{9}{|l|}{ Emotional } \\
\hline Low & 2 & 8.7 & & 2 & 8.7 & & & \\
\hline Medium & 2 & 8.7 & $75.00 \pm 21.3$ & 1 & 4.3 & $75.24 \pm .20 .62$ & .214 & .833 \\
\hline Good & 13 & 56.5 & & 14 & 60.9 & & & \\
\hline Perfect & 6 & 26.1 & & 6 & 26.1 & & & \\
\hline \multicolumn{9}{|l|}{ School function } \\
\hline \multicolumn{9}{|l|}{ Low } \\
\hline Medium & 0 & 0.0 & & 2 & 8.7 & & & \\
\hline Good & 15 & 65.2 & $58.34 \pm 12.17$ & 14 & 60.9 & $56.5 \pm .17 .20$ & 1.000 & .328 \\
\hline \multirow[t]{2}{*}{ Perfect } & 8 & 34.8 & & 6 & 26.1 & & & \\
\hline & 0 & 0.0 & & 1 & 4.3 & & & \\
\hline
\end{tabular}

Statistical Significant difference $\leq 0.05$ 
Table 3. Differences between children scores related to the depression, anxiety, and stress (DASS) scores pre and post health instruction sessions

\begin{tabular}{|c|c|c|c|c|c|c|}
\hline \multirow[t]{2}{*}{ DASS items } & \multicolumn{2}{|c|}{ Pre } & \multicolumn{2}{|c|}{ Post } & \multirow[t]{2}{*}{$\mathbf{t}$} & \multirow[t]{2}{*}{ p-value } \\
\hline & No & $\%$ & No & $\%$ & & \\
\hline \multicolumn{7}{|l|}{ Depression } \\
\hline normal & 0 & 0.0 & 0 & 0.0 & & \\
\hline mild & 0 & 0.0 & 5 & 21.7 & -3.761 & $.001 *$ \\
\hline moderate & 8 & 34.8 & 8 & 34.8 & & \\
\hline severe & 10 & 43.5 & 7 & 30.4 & & \\
\hline extremely severe & 5 & 21.7 & 3 & 13.0 & & \\
\hline \multicolumn{7}{|l|}{ Anxiety } \\
\hline normal & 0 & 0.0 & 0 & 0.0 & & \\
\hline mild & 0 & 0.0 & 0 & 0.0 & 3.867 & $.001 *$ \\
\hline moderate & 3 & 13.0 & 8 & 34.8 & & \\
\hline severe & 6 & 26.1 & 7 & 30.4 & & \\
\hline extremely severe & 14 & 60.9 & 8 & 34.8 & & \\
\hline \multicolumn{7}{|l|}{ Stress } \\
\hline normal & 0 & 0.0 & 0 & 0.0 & & \\
\hline mild & 8 & 34.8 & 15 & 65.2 & 4.596 & $.000^{*}$ \\
\hline moderate & 9 & 39.1 & 8 & 34.8 & & \\
\hline severe & 6 & 26.1 & 0 & 0.0 & & \\
\hline extremely severe & 0 & 0.0 & 0 & 0.0 & & \\
\hline
\end{tabular}

$*$ Statistical Significant difference $\leq 0.05$

Table 3: It is clear from this that there was differences between children scores on the depression pre and post health instruction which showed decline with highly statistical significant difference where $p=.001$. Also, there was differences between children scores on the anxiety and stress scores pre and post health instruction and showed statistical significant decline where $\mathrm{p}$ value $=.001$ and .000 respectively.

Table 4: this table presents the relation between the total peds QOL scores and children demographic and clinical data pre and post health instructions, According to the table, there was statistical significance relation between children age, study phase and their QOL scores pre health instruction where $\mathrm{p}=.032, .010$ respectively. As well as, there was no statistical significance relation between the total peds QOL scores and children demographic and clinical data in relation to all domains post health instruction where $\mathrm{p}$ value $>0.05$.

Table 4. Relation between the total quality of life (ped 4.0) scores and thalassemic children characteristics pre and post health instructions

\begin{tabular}{lcccc}
\hline Variables & \multicolumn{2}{c}{ Pre health instruction } & \multicolumn{2}{c}{ Post health instruction } \\
\cline { 2 - 5 } & \multicolumn{1}{l}{ Mean \pm SD } & P value & Mean \pm SD & P value \\
\hline Age (in years) & $60.40 \pm 11.75$ & .032 & $62.50 \pm .10 .27$ & .978 \\
$7-11$ & $64.75 \pm 6.65$ & & $63.53 \pm 10.25$ & \\
$\geq 12$ & & & & \\
Gender & $64.56 \pm .8 .67$ & .175 & $65.62 \pm .7 .45$ & .082 \\
Female & $60.41 \pm .11 .22$ & & $61.45 \pm 11.12$ & \\
Male & & & & \\
Study phase & & & & \\
Primary level & $54.16 \pm 10.83$ & .010 & $56.25 \pm .7 .65$ & .032 \\
Prep-school & $67.50 \pm 7.08$ & & $66.25 \pm .9 .85$ &
\end{tabular}


Secondary level

$64.00 \pm 5.97$

$68.75 \pm 8.82$

Thalassaemia family history

No

$58.75 \pm 10.50 \quad .061$

$60.75 \pm 9.57$

.213

Yes

$67.00 \pm 8.00$

$66.65 \pm 10.52$

Mother education

Illiterate

$60.40 \pm 7.90$

$60.0 \pm 8.40$

Read and write

$54.5 \pm 13.80$

.104

$59.25 \pm 7.85$

$\geq$ Secondary

$71.75 \pm 8.40$

$73.25 \pm 3.12$

\section{Father education}

Illiterate

$65.0 \pm 9.47$

$66.5 \pm .285$

Read and write

$56.25 \pm 6.67$

.088

$57.0 \pm 11.21$

$\geq$ Secondary

$60.5 \pm 12.8$

$62.5 \pm 8.82$

Blood transfusion Frequency

None

$65.5 \pm 10.8$

$64.0 \pm 13.7$

Once / month

$54.5 \pm 8.67$

.058

$59.25 \pm 6.67$

.340

Once/2 month

$61.25 \pm 8.12$

$60.0 \pm 12.9$

Once/6month

$68.75 \pm 9.7$

$68.75 \pm 7.9$

Length of diagnosis

$$
\begin{aligned}
& \leq \text { year } \\
& \leq \text { year }-5 \text { years } \\
& >5 \text { years }
\end{aligned}
$$$$
66.5 \pm 11.35
$$$$
.422
$$$$
59.5 \pm 10.3
$$$$
65.0 \pm 9.47
$$

$66.50 \pm 9.52$

$60.75 \pm 10.15$

$68.75 \pm 10.82$

Statistical Significant $\leq 0.05$

Table 5: this table describes the relation between the total DASS scores and children demographic and clinical data pre and post health instructions. It is evident that there was no statistical significance relation between the total depression, anxiety and stress scores and children demographic and clinical data pre and post health instructions where $\mathrm{p}$ value $>0.05$.

\begin{tabular}{|c|c|c|c|c|c|c|}
\hline \multirow[t]{2}{*}{ Variables } & \multicolumn{2}{|c|}{ Depression } & \multicolumn{2}{|c|}{ Anxiety } & \multicolumn{2}{|c|}{ Stress } \\
\hline & $\begin{array}{c}\text { Mean } \pm \text { SD } \\
\text { pre }\end{array}$ & $\begin{array}{c}\text { Mean } \pm \text { SD } \\
\text { post }\end{array}$ & $\begin{array}{c}\text { Mean } \pm \text { SD } \\
\text { pre }\end{array}$ & $\begin{array}{c}\text { Mean } \pm \text { SD } \\
\text { post }\end{array}$ & $\begin{array}{c}\text { Mean } \pm \text { SD } \\
\text { pre }\end{array}$ & $\begin{array}{c}\text { Mean } \pm \text { SD } \\
\text { post }\end{array}$ \\
\hline \multicolumn{7}{|c|}{ Age (in years) } \\
\hline $7-11$ & $23.7 \pm 3.2$ & $21.22 \pm 6.6$ & $23.0 \pm 6.3$ & $18.4 \pm 4.2$ & $23.4 \pm 4.8$ & $21.6 \pm 3.8$ \\
\hline$\geq 12$ & $22.8 \pm 34$ & $20.25 \pm 7.0$ & $22.3 \pm 7.4$ & $20.2 \pm 5.1$ & $24.1 \pm 4.3$ & $22.2 \pm 5.1$ \\
\hline$P$ value & .791 & .789 & .435 & .870 & .408 & .750 \\
\hline \multicolumn{7}{|l|}{ Gender } \\
\hline Female & $23.6 \pm 4.6$ & $21.2 \pm 4.8$ & $22.5 \pm 6.8$ & $17.3 \pm 2.3$ & $25.6 \pm 3.7$ & $20.4 \pm 2.1$ \\
\hline Male & $21.8 \pm 3.1$ & $21.1 \pm 3.3$ & $23.0 \pm 6.6$ & $19.3 \pm 3.4$ & $22.6 \pm 4.7$ & $20.9 \pm 3.7$ \\
\hline$P$ value & .946 & .861 & .867 & .976 & .143 & .941 \\
\hline
\end{tabular}

Table 5. Relation between the total DASS scores and children demographic and clinical data pre and post health instructions 


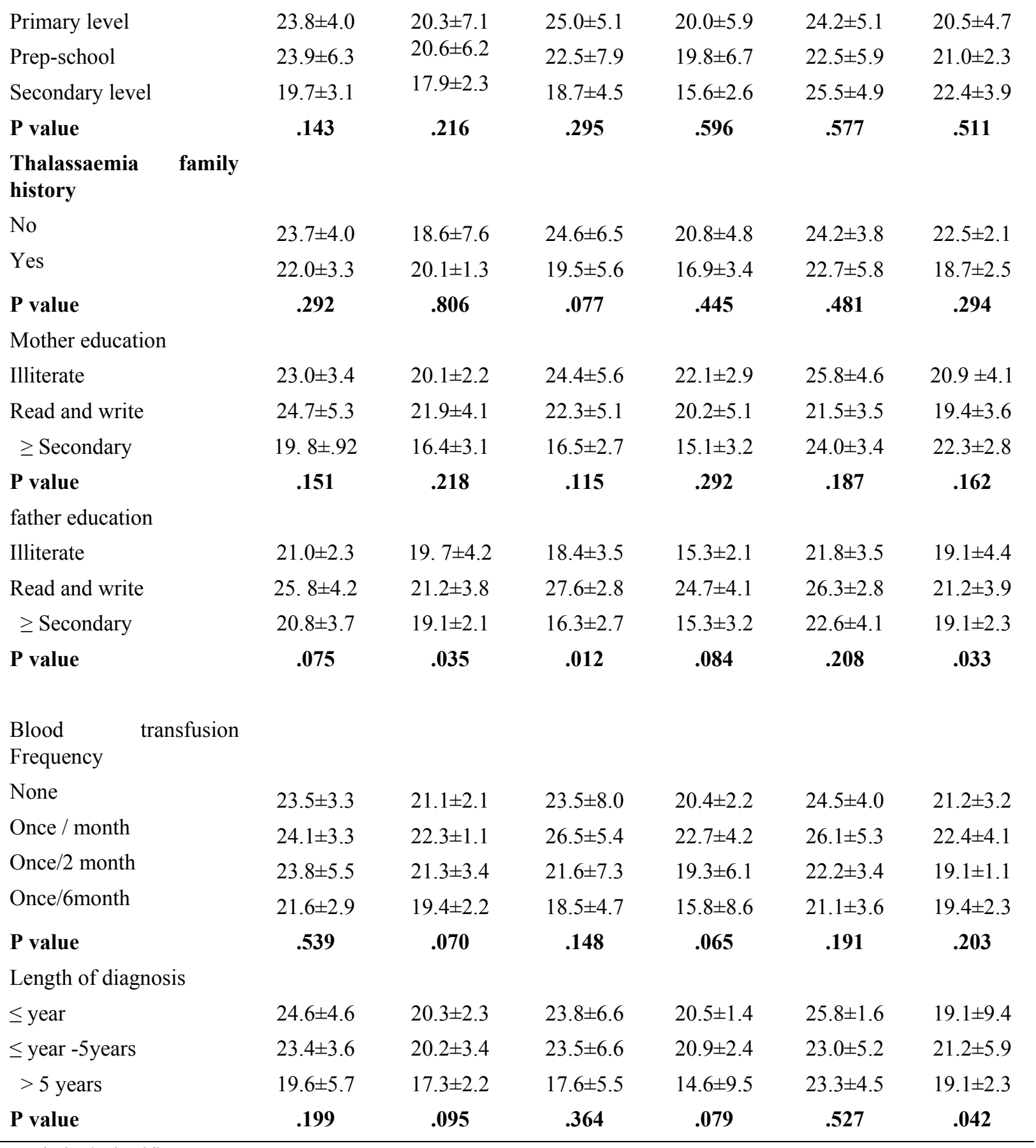

Statistical Significant $\leq 0.05$

\section{Discussion}

Beta thalassemia major is a chronic, genetically determined hematological disorder and has gained little investigation on the psychological aspects of the disease and quality of life adjustment of children with this disease. School age and adolescence itself is a time which demands more adjustment skills. An illness superimposed on the existing problems causes a physical and emotional outburst, which needs to be handled properly. If not, the overlooked needs may become manifest as psychological problems (Malevich et al., 2004). So far, only a few studies have been conducted analyzing the quality of life in $\beta$-thalassaemia (Baghianimoghadam et al. 2011; Elalfy et al, 2014). Knowledge of adaptive behaviors used by parents provides nurses with the possibility to design intervention programs for an appropriate support of them (Nikfarid et al., 2012). 
Concerning the socio-demographic characteristics of studied thalathemic children, finding of the present study revealed that more than two third of children in the age group ranged from 7 to 11 years old with mean age between $9.95 \pm 3.6$ years, and more than two third of them were males. These findings explained that the ratio of male coming to treatment was higher than female. This explanation is similarly with the findings of (Mostafa \& $\mathrm{Ab}$ Elaziz, 2014) who mentioned that more than half of the studied of thalathemic children were males at Zagazig university Hospitals. As well as, this finding goes in line with a finding of (Salama et al., 2006) that has done his study at Mansoura University Children's Hospital, Mansoura, in Egypt.

As regards family history of thalathemia, the present study revealed that more than two third of studied patients reported had previous history for thalthemia among their family members. This finding is in agreement with (Gharaibeh et al., 2009; Ammad et al., 2011) who found that more than two thirds of children had sick relatives and siblings with thalassemia. This may be due to increased consanguineous marriages in rural areas such as Beni Suef City.

Regarding blood transfusion frequency among studied children this study finding illustrated that a higher frequency of blood transfusion among thalassaemic children, where nearly two third of them was observed required blood transfusions once monthly or every two month in our study. This finding goes in line with (Aydinok et al.,2005) who found that $33 \%$ and $93 \%$ thalathemic children in a private and government setting respectively needed blood transfusion more than once a month. As well as, this finding goes in line with (Ismail et al., 2013) who assessed the health-related quality of life in Malaysian children with thalassemia and found that $66 \%$ of thalathemic children required blood transfusion monthly.

The current study illustrated that based on the application of DASS scale among children to assess their psychological problems, the study findings showed that nearly half of studied children with thalassemia had severe depression and two third of them suffering from extremely severe anxiety and more than one third had moderate stress. These findings were consistent with results of (Shaligram et al., 2007) who done his study at national institute of mental health and neurosciences, Bangalore and found that forty four percent of the children with thalassemia had psychological problems, anxiety symptoms and depression were the main findings These findings also supported by (Behdani et al., 2015) who done his study in Iran about psychological aspects in children and adolescents with thalassemia and reported that children with thalassemia major have more psychological problems particularly depression, anxiety, than healthy ones. In the other hand these findings is contraindicated with (Aydinok et al., 2005; Khani et al., 2007) who found that most children with thalassemia had no psychological problems. However, illness-induced anxieties and depression among these patients had been well-documented in many studies (Roy\& Chatterjee, 2007; Gharaibeh et al., 2007; Ammad et al., 2011). This findings may explained by a fear of chronic diseases like thalassemia that do not have a definite cure, cost of treatment and unavailability of treatment can be one of the important factors leading to these psychological reactions. This explanation is supported by (Koutelekos \& Haliasos, 2013) who stated that in developing countries where is observed lack of public awareness or the cost of therapy is either too expensive or not available, helpless thalassemic children and their parents at their effort to cope with the disease may experience severe emotional difficulties, such as frustration, sadness, hostility, depression, anxiety, fear of death, lack of confidence, isolation and anger.

Meanwhile, the differences between children scores on the anxiety and stress scores pre and post health instruction showed significant decline. These results explained that providing information to children and their caregivers consider the way for ventilation and expressing their feelings and needs. Also provision of accurate information to thalasssemic individuals about the therapeutic regimen by expertise health professionals and nurses is a key-factor that plays a vital role on the outcome of psychological problems. This explanation supported by(Koutelekos \& Haliasos, 2013) who stated that, providing information to patients in understandable and accurate terms about the nature of the disease, the need for treatment, the new medical protocols, has beneficial effects both on the outcome of thalassemia and psychological problems as depression. Knowledge deficits may result in unnecessary depression.

Concerning the quality of life of studied children with thalassaemia, the findings of this study demonstrated that, according to ped QOL scale more than two third of thalassemic children had medium grade in physical functioning This finding in agreement with the results reported by (Ismail et al, 2013) who assessed the health-related quality of life in Malaysian children with thalassemia, who found that thalassaemia has a negative impact on perceived physical functioning. Similarly, (Caocci et al, 2012) reported in a cohort of thalassemia patients from Syria, Palestine, and Iraq that reduction in the physical functioning as aresult of frequency of blood transfusion and iron chelation. This finding goes in line with study done by (Mostafa \& Ab Elaziz, 2014) at Zagazig University who found that more than half of children didn't practice any kind of play because of physical weakness and feeling of exhausted. As well as, in accordance with (Khurana et al., 2006) who studied 
the psychosocial life aspects of Indian adolescents suffering from $\beta$-thalassemia major and reported that over two thirds of the adolescents were unable to engage in outdoor play at the same level as their peers, because of physical weakness related to their disease. Also, (Caro et al., 2002) who found that, less than one quadrant of conventionally treated thalassemia major patients had their activities very often stopped due to thalassemia, its complications or desferrioxamine treatment, and $20 \%$ had their physical activities limited at least a bit. This finding may be due anemia and complications of iron overload this explanation supported by (Cheuk et al, 2008) who stated that the regular period of mild anemia before the scheduled of transfusion which might limit their physical capacity as thalassemia leads to low hemoglobin level resulting in fatigue and general weakness.

In the present study, more than one third of thalathemic children reported good social relationship. This result may be explained by that in rural area especially in Egypt there are strong relationship between families and most of them were relatives as well as support by friends and family members has been a key factor in maintaining a good relationship among thalathemic children. Similar results also showed in a study conducted by (Amoudi et al, 2014) in Western Saudi who found that in their study about quality of life among patient with beta-thalassemia major in western Saudi Arabia as for the social functioning score, and stated that our sample averaged a score of 75 , which is significantly higher and explained that and attribute this to the presence of more social contacts and circles, family and friends.

In addition, the present study results indicated that more than half of studied children with thalassemia had good grade in their quality of life in emotional functioning as fear, anger as reported by children and their caregivers. These findings disagreed with (Pakbaz et al., 2005) who found that emotional functioning is one of the weaker domains among thalassaemia children as they experience fear and concerns over their health status. On the other hand, only one third scored good grade in school functioning. This finding confirmed by (Ismail et al., 2006) who mentioned that the school functioning among studied children with thalassemia scored the lowest subscale. This finding can attributed by that children with thalassemia requiring regular blood transfusion in hospital and resulting in absent of children from school. As well as disease itself affect on their general health. This finding goes in line with (Ismail et al. 2013) who found that the school functioning score $(50.59 \pm 15.31)$ was the lowest of the psychosocial measure, followed by emotional functioning $(59.92 \pm 16.83)$ and social functioning (78.01 \pm 13.92$)$. This may be explained by that the school functioning domain was the most affected domain. Similar to reported data school problems were common and found in all thalassemic patients because of frequent hospitalization, school absenteeism, and poor peer group adjustment and disease related complications. Unfortunately, findings of this study documented that there was no significant difference observed in grades of children HRQOL post health instruction sessions.

Concerning the relationship between total score of HRQOL and socio- demographic and clinical data, these study findings showed that there was minimal statistical relationship between children age, study phase and their peds QOL scores. As well as, there was no statistical significance relation between the total peds QOL scores and children demographic and clinical data. These study findings confirmed by (Elalfya et al.,2014) who found that the sex difference had no significant impact on QOL scores in any of the domains, which is in agreement with other studies done by (Thavorncharoensap et al., 2010).On the other hand the results of this study demonstrated that there was no statistical significance relation between the total depression, anxiety and stress scores with children demographic and clinical data pre and post health instruction sessions where p value $>0.05$, this result was in agreement with (Shaligram et al., 2007) who found that there was no correlation between the psychological problems in the children with thalassemia and their clinical variables and demographic data except for sex of the children.

Our study further findings indicated that children with thalthemia had psychological problems with poorer quality of life. Meanwhile following health instructions there was significant improvement in children psychological aspects. A recently published study highlighted the significant negative impact of thalassaemia on psychosocial functioning among Thai children. More psychological support should be provided to thalassaemia patients while in-depth studies involving both children and parents would establish correlations between individual psychological outcomes and health related quality of life (Ammad et al., 2011). Also, it corresponds to the results of (Shaqaqi et al., 2009) who stated that holding of family educational classes had been effective on the health of parents with mentally retarded children and improves the level of physical disorders and anxiety and public depression and health.

\section{Conclusion}

Our study reflects that thalassaemia has a negative influence on studied children psychological aspect causing anxiety and depression. As well as, it resulting in poorer in their health related quality of life, especially in the 
physical and school functioning domains. However, there is positive association between health instructions sessions and depression, anxiety and stress scores among children with thalassaemia which showed great decline, this explained the importance of education, ventilation and counseling with children and their parents according to their health needs. As well as, no improvement showed in their health related quality of life. Therefore, better health education programs for children and their parents has great impact on their psychological problems. The research findings for this study indicate the need for systemic education for children and caregivers to improve their health related quality of life.

\section{Recommendation}

According to findings of this study, we recommended that:

- Provision of information by nurses for thalassamic children should be individualized and appropriate to the children age, developmental stage and their needs.

- It is necessary to develop educational unit in every hospital for chronically ill children especially children with thalassemia.

- Regular health educational programs should be done with collaboration of all health team in hematological unit.

- Pediatric and psychiatric nurses should be cooperate with them to apply comprehensive educational program for children and their families to deal with their health problems and psychological problems related to thalassaemia

- Further researches are needed to investigate various coping strategies to improve thalassaemic children health related quality of life.

\section{Acknowledgements}

The authors gratefully acknowledge the support and the collaborating of head of hematological department and nurses and nurses interns who help us in this study. The authors also thank children and their parent's contribution and participation.

\section{References}

Ammad, S.A., Mubeen, S.M., \& Shah, S.F. (2011, May). Parents' opinion of quality of life (QOL) in Pakistani thalassaemic children. J Pak Med Assoc., 61(5), 470-3.

Amoudi, A.S., Balkhoyor, A.H., Abulaban, A.A., Azab, A.M., Radi, S.A., \& Ayoub, M.D., et al. (2014). Quality of life among adults with beta-thalassemia major in western Saudi Arabia. Saudi Medical Journal, 35(8), 882-5.

Aydinok, Y., Erermis, S., Bukusoglu, N., Yilmaz, D., \& Solak, U. (2005, February). Author information Psychosocial implications of Thalassemia Major. Pediatr Int., 47(1), 84-9.

Azarkeivan, A., Hajibeigi, B., S.M., Lankarani, M.M., \& Assari, S. (2009). Associates of poor physical and mental health-related quality of life in beta-thalassemia major/intermedia. J Res Med Sci., 14, 349-55.

Baghianimoghadam, M.H., Sharifirad, G., Rahaei, Z., Baghianimoghadam, B., \& Heshmati, H. (2011). Health related quality of life in children with thalassemia assessed based on SF-20 questionnaire in Yazd, Iran: a case-control study. Cent Eur J Public Health, 19, 165-169.

Behdani, M., Zeinali, S., Khanahmad, H., Karimipour, M., Asadzadeh, N., Azadmanesh, K., \& Khabiri, A. (2015, June). Psychological Aspects in Children and Adolescents with Major Thalassemia: A Case-Control Study. Iran J Pediatr., 25(3), e322.

Borgna - Pignatti,.C, \& Gamberini, M.R. (2011). Complications of thalassemia major and their treatment. EXP Rev Hematol., 4, 353-66.

Caocci, G., Efficace, F., Ciotti, F., Roncarolo, M.G., Vacca, A., \& Piras, E., et al. (2012). Health related quality of life in Middle Eastern children with beta-thalassemia. BMC Blood Disord., $12,6$. https://doi.org/10.1186/1471-2326-12-6

Caro, J.J., Ward, A., \& Green, T.C, et al. (2002). Impact of thalassemia major on patients and their families. Acta Haematol, 107, 150-57. https://doi.org/10.1159/000057633

Chaturvedi, S. K., Girimaji, S. C., \& Shaligram, D. (2007). Psychological problems and quality of life in

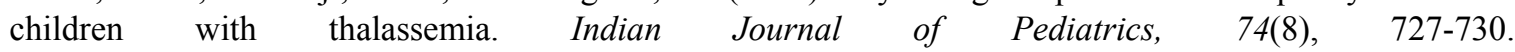
https://doi.org/10.1007/s12098-007-0127-6 
Cheuk, D.K., Mok, A.S., \& Lee, A.C. (2008). Quality of life in patients with transfusion-dependent thalassemia after hematopoietic SCT. Bone Marrow Transplantation, 42, 319-327. https://doi.org/10.1038/bmt.2008.165

Economou, M., Zafeiriou, D.I., Kontopoulos, E., Gompakis, N., Koussi, A., \& Perifanis, V. (2006). Athanasiou-Metaxa M:Neurophysiologic and intellectual evaluation of beta-thalassemia patients. Brain Dev., 28, 14-18. https://doi.org/10.1016/j.braindev.2005.03.006

Elalfya, S. M., Faridb, M. N., Labiba, J. H., \& RezkAllahc, H. K. (2014). Quality of life of Egyptian $\beta$-thalassemia major children and adolescents. Egyptian Journal of Haematology, 39, 222-226. https://doi.org/10.4103/1110-1067.153963

El-Beshlawy, A. (2009). Prevention of Hemoglobinopathies in Egypt. 1st Pan-Eastern Conference on Heamoglobinopathies. Retrieved 12 March 2010 from http://www.thalassaemia.org.cy/pdf/syria/04.pdf

Gharaibeh, H., Amarneh, B., \& Zamzam, S. (2009). The psychological burden of patients with beta Thalassemia major in Syria. Pediatrics International, 51, 630-636. https://doi.org/10.1111/j.1442-200X.2009.02833.x

Indaratna, K., Nuchprayoon, I., Riewpaiboon, A., Thavorncharoensap, M., Torcharus, K., \& Ubol, B. (2010). Factors affecting health-related quality of life in Thai children with thalassemia. Blood Disorders, 10(1). https://doi.org/10.1186/1471-2326-10-1

Ismail, M., Chun, C.Y., Yusoff, N.A.M., Shahar, S., \& Manaf, Z.A. (2013). Quality of Life among Thalassaemia Children, Adolescent and Their Caregivers. SainsMalaysiana, 42, 373-80.

Ismail. A., Campbell, M.J., Ibrahim, H.M., \& Jones, G.L. (2006). Health related quality of life in Malaysian children with thalassemia. Health Qual Life Outcomes, 4, 39. https://doi.org/10.1186/1477-7525-4-39

James W. Varni. (2006). PedsQL Scales and Modules. MAPI Research Institute in Lyon, France. Retrieved from eprovide.mapi-trust.org

Johnson, J.Y. (2010). Pediatric Nursing Demystified. McGraw-Hill Co, United States, p.142.

Khani, H., Majdi, M.R., Montazeri, A., Hosseini, S.H., Ghorbani, A., \& Ramezani, M., et al. (2007). Quality of life in Iranian Beta-thalassemia major patients of southern coastwise of the Caspian Sea. Journal of Behavioral Sciences, 8(35-36), 325-32.

Khurana, A., Katyal, S., \& Marwaha, R.K. (2006). Psychosocial Burden in Thalassemia. Indian Journal of Pediatrics, 73(10), 877-880. https://doi.org/10.1007/BF02859278

Koutelekos, J., \& Haliasos, N. (2013). Depression and Thalassemia in children, adolescents and adults. Health Science Journal, 7(3).

Mahmoud, M.H. (2009). Thalassemia around the World: Egypt. Retrieved 14 March 2010 from http://www.thalassemia.org/index.php?option=com_content\&view=article\&id=222:thalassemia-aroundtheworld-egypt\&catid=1:latest-news

Manevich, T.M., Sokolova, E.D., Lakhno, N.N., \&Rogovina, E.G. (2004). Specifics of personality and mental status in children and adolescence with chronic tension -type headache. Zh Nevrol Psikhiatr Im S S Korsakova, 104, 11-5.

Mazzone, L., Battaglia, L., Andreozzi, F., Romeo, M.A., \& Mazzone, D. (2009). Emotional impact in beta-thalassaemia major children following cognitive-behavioural family therapy and quality of life of caregiving mothers. Clin Pract Epidemiol Ment Health, 5, 5-10.

Monastero, R., Monastero, G., Ciaccio, C., Padovani, A., \& Camarda, R. (2000). Cognitive deficits in $\begin{array}{lllll}\text { beta-thalassemia } \text { major. Acta Neurol } & \text { Scand. }\end{array}$ https://doi.org/10.1034/j.1600-0404.2000.102003162.x

Mostafa, S. M., \& Ab Elaziz, M. (2014). Factors Affecting Compliance Plan of Thalassemia Children and their Mothers in Outpatient Clinic at Zagazig University Hospitals. Journal of Biology, Agriculture and Healthcare, 4(3).

Nikfarid, L., Eezadi, H., \& Shakoori, M. (2012). Coping Behaviors of Mothers of Chronically Ill Children. IJN., 24(74), 53-62.

Pakbaz, Z., Treadwell, M., Yamshita, R., Quirolo, K., Foote, D., Quill, L., ... Vichinsky, E.P. (2005). Quality of life in patients with thalasssemia intermediate compared to thalassemia major. New York Academy of Sciences, 1054, 457-461. 
Roy, T., \& Chatterjee, S.C. (2007). The experiences of adolescents with thalassemia in West Bengal, India. Qual Health Res., 17, 85-93. https://doi.org/10.1177/1049732306296400

Salama O.S., AL-Tonbary, A.Y., Shahin, A.R., et al. (2006). Unbalanced bone turnover in children with beta thalassemia. Hematology, 11(3), 197-202. https://doi.org/10.1080/10245330600702851

Salehi, S., \& Morovati, S. (2016). Coping behaviors in families with children suffering from thalassemia major and evaluating the implementation effect of nursing intervention on these behaviors. International Journal of Medical Research \& Health Sciences, 5(2), 58-63.

Shaligram, D., Girimaji, S.C., \& Chaturvedi, S.K. (2007). Psychological problems and quality of life in children with thalassemia. Indian J. Pediatr., 74, 727-30. https://doi.org/10.1007/s12098-007-0043-9

Shaqaqi, F., Kakojuybari, A.A., \& Salami, F. (2009). The family education effect on psychological health of parents with educable mentally retarded children. Journal of Behavioral Sciences, 58-69.

Taouk, M., Lovibond, P.F., \& Laub, R. (2008). Psychometric Properties of an Arabic Version of the Depression Anxiety Stress Scale (DASS). Retrieved from http://www.psy.unsw.edu.au/Groups/Dass/Arabic/htm

Telfer, P., Constantinidou, G., Andreou, P., Christou, S., Modell, B., \& Angastiniotis, M. (2005). Quality of life in thalassaemia. Ann. NY Acad. Sci., 1054, 273-282. https://doi.org/10.1196/annals.1345.035

Thavorncharoensap, M., Torcharus, K., Nuchprayoon, I., Riewpaiboon, A., Indaratna, K., \& Ubol, B.O. (2010). Factors affecting health-related quality of life in Thai children with thalassemia. BMC Blood Disord., 10, 1-10. https://doi.org/10.1186/1471-2326-10-1.

Torcharus, K., \& Pankaew, T. (2011). Health-related quality of life in thalassemia treated with iron chelation. Royal Thai Army Medl J., 64, 3-10.

WHO. (2008). Management of Haemoglobin Disorders: Report of a joint WHO-TIF meeting. Geneva: World Health Organization.

Wong, L., George, E., \& Tan, J.A. (2011). Public perceptions and attitudes toward thalassaemia: Influencing factors in a multi-racial population. BMC Public Health, 11, 193. https://doi.org/10.1186/1471-2458-11-193

\section{Copyrights}

Copyright for this article is retained by the author(s), with first publication rights granted to the journal.

This is an open-access article distributed under the terms and conditions of the Creative Commons Attribution license (http://creativecommons.org/licenses/by/4.0/). 